\section{Molecular Syndromology}

Mol Syndromol 2016;7:299-301

DOI: $10.1159 / 000450807$
Accepted: September 12, 2016

\title{
Discovering Patterns of Structural Variation by Mining Molecular Fossils
}

Structural genome variations (SVs) are defined as changes in the organization of DNA sequence elements involving at least $50 \mathrm{bp}$, while smaller structural genome changes are known as indels [Alkan et al., 2011]. Conventionally, SVs are classified according to the number of chromosome breaks by which they originated as either copy number variations (CNVs) consisting of terminal deletions and duplications (1-break events), interstitial deletions and duplications (2-break events), or as complex chromosome rearrangements involving more than 2 breaks [Poot and Haaf, 2015]. CNVs larger than $100 \mathrm{~kb}$ arise de novo at an estimated rate of $\sim 1.2 \times 10^{-2} \mathrm{CNVs}$ per meiosis [Itsara et al., 2010]. All types of germline SVs together occur more frequently than germline single nucleotide variations (SNVs), affect more nucleotides, and may have a greater phenotypic impact than SNVs [Stankiewicz and Lupksi, 2010; Campbell and Eichler, 2013]. In up to $20 \%$ of children with developmental and/ or intellectual delay, phenotypically significant SVs can be detected upon investigation by both classical karyotyping and array CGH [Hochstenbach et al., 2009; Cooper et al., 2011]. This underscores the need for comprehensive analyses of SVs in the human genome. Thousands of human genomes have been analyzed by classical karyotyping, FISH, and genome-wide array CGH. While this provided information regarding the structure of the SVs, their breakpoints could not be resolved at nucleotide resolution. For the latter, paired-end and mate-pair sequencing has been used [Kloosterman et al., 2012]. The infor- mation thus obtained allows inferring the possible mechanism(s) of origin of the SVs [Kloosterman et al., 2012; Poot and Haaf, 2015]. Since these analyses are laborious, they have been performed only on a case-by-case basis for selected individuals, which limits the number of cases analyzed and may have introduced ascertainment biases. Recently, a converse approach has been taken. SVs were identified by comparing sequence data from whole genome sequencing projects of healthy individuals derived from the general population with the human reference genome sequence. All deviations from the human reference genome sequence can be considered as 'molecular fossils' representing SVs. These studies not only provided unprecedented insights into the mechanism(s) of origin of CNVs, inversions, and indels, but also an estimate of their relative frequency in the general population.

To distinguish nonallelic homologous recombination (NAHR), with long stretches of homology surrounding the breakpoints, from transposable element insertions (TEI), with short homologies containing mobile elements close to the SV, from nonhomologous end-joining (NHEJ), with little or no homology at breakpoints, and from template-switching mechanisms during replication (FoSTeS), Abyzov et al. [2015] analyzed 8,943 deletion breakpoints in 1,092 whole genome sequences. All breakpoints were associated with evolutionarily less-conserved regions, spanning up to hundreds of kilobases downstream and upstream from the breakpoints of the SVs. TEI breakpoints were 5 times more likely to reside in hy-

\section{KARGER}

E-Mail karger@karger.com

www.karger.com/msy
(C) 2016 S. Karger AG, Basel

$1661-8769 / 16 / 0076-0299 \$ 39.50 / 0$
Martin Poot

Department of Human Genetics, University of Würzburg

Biozentrum, Am Hubland

DE-97074 Würzburg (Germany)

E-Mail Martin_Poot@hotmail.com 
pomethylated regions than expected by chance, which suggests preferential TEI in transciptionally active chromatin regions. Breakpoints with an NAHR signature were associated with open, early replicating chromatin, as was evident from the high density of histone $\mathrm{H} 3$ lysine 4 and lysine 79 dimethylation, and lysine 9 acetylation [Abyzov et al., 2015]. In contrast, sites with NHEJ were depleted for these histone marks. The authors hypothesize that NAHR deletions may occur in embryonic and germline cells in the absence of DNA replication and cell division. In contrast, NHEJ-associated breakpoints often showed sequence microinsertions, which were templated from later replicating genomic sites spaced at 2 characteristic distances from the breakpoint. These microinsertions were consistent with FoSTeS-like events. Such microinsertions, ranging from 1 to $96 \mathrm{bp}$ in length with the majority being less than $10 \mathrm{bp}$, were found in association with 2,391 (27\%) of all deletions. NHEJ-mediated deletions are typically associated with later replicating chromatin regions, while TEIs show no significant relationship to replication time.

The association of NAHR with early replication timing is surprising, since NAHR is supposed to occur during meiotic cell division as a requirement for generating germline SVs. In addition to the expected association of NAHR breakpoints with higher recombination rates, higher GC content and higher density of CpG motifs, they were also associated with open chromatin and active histone marks in mitotically dividing cells. At the time of chromosome segregation, there is no defined DNA structure, histone marks have been erased, and DNA replication has been completed. The authors hypothesize that some of the NAHR-mediated SVs may have arisen in the germline and during early embryogenesis in the absence of DNA replication and cell division. Open and transcriptionally active, hypomethylated chromatin contains unpacked easily melting, probably single-stranded DNA. Such DNA may serve as a template for double-strand break repair in homologous region(s) in the same chromosome. Thus, intramolecular NAHR, i.e., homologous recombination between regions in the same chromosome, would take place and generate a deletion as well as a piece of extra-chromosomal circular DNA, which would subsequently be lost.

By analyzing whole genome sequence data from 2,504 individuals, 42,279 biallelic deletions, 6,025 biallelic duplications, 2,929 multi-allelic CNVs, 786 inversions, 168 nuclear mitochondrial insertions, and 16,631 mobile element insertions (L1, SINE-R, VNTR, and Alu) were discovered [Sudmant et al., 2015]. Some of the deletions showed unexpected complexity. The breakpoints of $6 \%$ of the deletions overlapped with another deletion with distinct breakpoints, and 16\% had additional inserted sequences at their deletion breakpoints. Of the deletions with an insertion of at least $10 \mathrm{bp}, 30.3 \%$ had a recognizable duplicated sequence interval within the respective inserted sequence, and $12 \%$ had 2 or more apparent sequence duplications at the deletion breakpoints. These complex deletions could have resulted from templateswitching mechanisms, since microhomologies were typically observed between the breakpoints of deletions and the boundaries of insertion templates. The majority of inversions (54\%) corresponded to inverted duplications. The remaining inversions comprised 'inv and del' events, multiple-deletion events with inverted spacers, and highly complex sites. The apparent inversion complexity is most likely due to a process that forms complex SVs, potentially involving DNA replication errors, rather than due to recurrent rearrangements.

Taken together, SVs were enriched for eQTLs and in strong linkage disequillibrium with genome-wide association study (GWAS) hits [Schlattl et al., 2011; Sudmant et al., 2015]. Conversely, GWAS haplotypes were enriched up to 3-fold for common SVs, which emphasize the relevance of ascertaining SVs in GWAS-based disease studies. In addition, the large number of rare SVs found may be of value for rare variant-based disease association studies. The not earlier described patterns of SV complexity, particularly of inversions, indicate that other more complex mutational processes apart from nonallelic homologous recombination, retrotransposition, and NHEJ may have taken part in shaping our genome. Nevertheless, it remains difficult to fully disentangle the contributions of SV mutation rates and selective forces to the observed SVs.

Both studies demonstrate a high frequency of all classes of SVs in healthy individuals and indicate that our genome is much more plastic than previously thought [Abyzov et al., 2015; Sudmant et al., 2015]. This plasticity most likely results from genome maintenance mechanisms, which have previously been associated with the so-called 'genomic instability syndromes' but may have a much wider impact [Poot and Haaf, 2015]. The plasticity of our genome may also interfere with attempts to identify disease loci by GWAS. Thus, more detailed studies of the molecular mechanisms of SVs, e.g., mate-pair and paired-end sequencing, in both patients and healthy individuals are needed to assist in distinguishing 'natural' variation form true disease association.

Martin Poot 


\section{References}

Abyzov A, Li S, Kim DR, Mohiyuddin M, Stütz AM, et al: Analysis of deletion breakpoints from 1,092 humans reveals details of mutation mechanisms. Nat Commun 6:7256 (2015).

Alkan C, Coe BP, Eichler EE: Genome structural variation discovery and genotyping. Nat Rev Genet 12:363-376 (2011).

Campbell CD, Eichler EE: Properties and rates of germline mutations in humans. Trends Genet 29:575-584 (2013).

- Cooper GM, Coe BP, Girirajan S, Rosenfeld JA, $\mathrm{Vu} \mathrm{TH}$, et al: A copy number variation morbidity map of developmental delay. Nat Genet 43:838-846 (2011).
Hochstenbach R, van Binsbergen E, Engelen J, Nieuwint A, Polstra A, et al: Array analysis and karyotyping: workflow consequences based on a retrospective study of $36,325 \mathrm{pa}$ tients with idiopathic developmental delay in the Netherlands. Eur J Med Genet 52:161-169 (2009).

Itsara A, Wu H, Smith JD, Nickerson DA, Romieu I, et al: De novo rates and selection of large copy number variation. Genome Res 20: 1469-1481 (2010).

Kloosterman WP, Tavakoli-Yaraki M, van Roosmalen MJ, van Binsbergen E, Renkens I, et al: Constitutional chromothripsis rearrangements involve clustered double-stranded DNA breaks and nonhomologous repair mechanisms. Cell Rep 1:648-655 (2012).
Poot M, Haaf T: Mechanisms of origin, phenotypic effects and diagnostic implications of complex chromosome rearrangements. Mol Syndromol 6:109-133 (2015).

Schlattl A, Anders S, Waszak SM, Huber W, Korbel JO: Relating CNVs to transcriptome data at fine resolution: assessment of the effect of variant size, type, and overlap with functional regions. Genome Res 21:2004-2013 (2011).

Stankiewicz P, Lupski JR: Structural variation in the human genome and its role in disease. Annu Rev Med 61:437-455 (2010).

Sudmant PH, Rausch T, Gardner EJ, Handsaker RE, Abyzov A, et al: An integrated map of structural variation in 2,504 human genomes. Nature 526:75-81 (2015). 